University of Wollongong

Research Online

2010

The Clinical Services Redesign Program in New South Wales : perceptions of senior health managers

Malcolm R. Masso

University of Wollongong, mmasso@uow.edu.au

Glenn Robert

King's College, London, UK

Grace McCarthy

University of Wollongong, gracemc@uow.edu.au

Kathy Eagar

University of Wollongong, keagar@uow.edu.au

Follow this and additional works at: https://ro.uow.edu.au/ahsri

Research Online is the open access institutional repository for the University of Wollongong. For further information contact the UOW Library: research-pubs@uow.edu.au 


\title{
The Clinical Services Redesign Program in New South Wales : perceptions of senior health managers
}

\begin{abstract}
OBJECTIVE: This study explores the views of senior managers regarding their experience of participating in the Clinical Services Redesign Program (CSRP) in New South Wales and the impact of that Program. METHODS: Semi-structured interviews were conducted in 2007 with 42 senior managers working in the NSW health system. RESULTS: Managers reported being increasingly oriented towards efficiency, achieving results and using data to support decision-making. The increased focus on managing performance was accompanied by concerns about the narrowness of the indicators being used to manage performance and how these are applied. The value placed by interviewees on the use of 'competition' as a lever for improving services varied. Leadership was repeatedly identified as important for long-term success and sustainability. No one was confident that the CSRP had yet been sufficiently embedded in day to day practice in order for it to keep going on its own. CONCLUSION: Our findings are generally consistent with the extensive literature on change management, performance management and leadership. Some cultural change has taken place in terms of observed patterns of behaviour but it is unrealistic to think that CSRP can on its own deliver the desired deeper cultural changes in the values and assumptions underpinning the NSW Health system. There is some evidence of dysfunctional aspects of performance management but no call for the focus on performance or redesign to be abandoned.
\end{abstract}

\section{Keywords}

senior, perceptions, wales, south, program, redesign, managers, services, health, clinical

\section{Publication Details}

M. R. Masso, G. Robert, G. McCarthy \& K. M. Eagar, "The Clinical Services Redesign Program in New South Wales: perceptions of senior health managers", Australian Health Review, 34(3), 2010, 352-359. 


\title{
The Clinical Services Redesign Program in New South Wales: perceptions of senior health managers
}

\author{
Malcolm Masso ${ }^{1,4}$ RN, BSc(Econ), MNA, MPH, Senior Research Fellow \\ Glenn Robert ${ }^{2}$ BA, MSc, PhD, Senior Research Fellow \\ Grace McCarthy ${ }^{3}$ BA, MA, PGDipLib, MBA, PhD, Lecturer \\ Kathy Eagar ${ }^{1} \mathrm{PhD}$, Professor and Director \\ ${ }^{1}$ Centre for Health Service Development, University of Wollongong, NSW 2522, Australia. \\ ${ }^{2}$ National Nursing Research Unit, King's College London, Strand, London, WC2R 2LS, UK. \\ ${ }^{3}$ Sydney Business School, University of Wollongong, NSW 2522, Australia. \\ ${ }^{4}$ Corresponding author. Email: mmasso@uow.edu.au
}

\begin{abstract}
Objective. This study explores the views of senior managers regarding their experience of participating in the Clinical Services Redesign Program (CSRP) in New South Wales and the impact of that Program.

Methods. Semi-structured interviews were conducted in 2007 with 42 senior managers working in the NSW health system.

Results. Managers reported being increasingly oriented towards efficiency, achieving results and using data to support decision-making. The increased focus on managing performance was accompanied by concerns about the narrowness of the indicators being used to manage performance and how these are applied. The value placed by interviewees on the use of 'competition' as a lever for improving services varied. Leadership was repeatedly identified as important for long-term success and sustainability. No one was confident that the CSRP had yet been sufficiently embedded in day to day practice in order for it to keep going on its own.

Conclusion. Our findings are generally consistent with the extensive literature on change management, performance management and leadership. Some cultural change has taken place in terms of observed patterns of behaviour but it is unrealistic to think that CSRP can on its own deliver the desired deeper cultural changes in the values and assumptions underpinning the NSW Health system. There is some evidence of dysfunctional aspects of performance management but no call for the focus on performance or redesign to be abandoned.
\end{abstract}

What is known about the topic? There has been growing interest internationally in the potential of industrial process improvement models (such as business process re-engineering, Six Sigma and Lean Manufacturing) to secure sustained improvements in the efficiency of healthcare services. Such approaches are often accompanied by the implementation of a rigorous performance management system. However, overall results in the healthcare sector have been mixed with outcomes sometimes falling short of stated ambitions. To date, in-depth research into the use of such approaches and systems in Australia has been limited.

What does this paper add? This paper reports on research in New South Wales to evaluate one such approach: the 3-year Clinical Services Redesign Program that aims to achieve transformational, sustainable, system-wide change by 'undertaking deep seated structural and cultural reform of traditional work practices'. The original CSRP business case envisaged a radical - rather than incremental - approach to system change, in keeping with a 're-engineering' ethos. The qualitative findings presented here are based on interviews in 2007 with 42 senior health managers working at different levels of the health system. These interviews explored the experience of participating in the CSRP and elicited views as to the perceived impact of the Program from a managerial perspective. The findings are related to theories of system level change and compared with the emerging evidence-base relating to large-scale improvement strategies in healthcare.

What are the implications for practitioners? Managers support the principle of managing performance by setting targets, with concerns primarily about the narrow focus of the selected targets, how the targets are applied locally and the nature of their central monitoring. Targets need to be well defined and measure the processes and outcomes that really matter. The principle of linking performance with service redesign was also supported. However, interviewees did not believe that changing culture to achieve sustainable change could be brought about by a single centrally-led change program. Significantly, leadership was seen as a critical factor in improving performance but needs to be considered within a broad framework (i.e. a system of leadership) that relies on more than just the attributes of individuals. Finally, management 
development should not be overlooked, or seen as less important than leadership development. Improvement projects frequently fail in implementation and this is as much a management issue as a leadership issue.

\section{Introduction}

There are many international examples of transformational ambition for healthcare services. ${ }^{1-3}$ Evidence is emerging about how to attain that ambition, while recognising that the process is complex, difficult and likely to be time consuming. ${ }^{4}$ The key issue is not whether the potential for more efficient, safer, more value-adding healthcare exists, but how to realise that potential across an entire healthcare system. The challenge facing policy-makers and managers is whether their current improvement strategies and approaches are enough to deliver that different future. ${ }^{5}$

In this wider context, the New South Wales Department of Health (henceforth referred to as the Department) has since 2004-05 been implementing a three-pronged reform agenda to improve the performance of the NSW health system with:

- a significant increase in resources (particularly additional hospital beds);

- top down performance management; and

- a formal Clinical Services Redesign Program (CSRP).

This agenda took place against a backdrop of organisational restructuring after a long period of relative stability involving abolition of all area health service boards and amalgamation into eight larger area health services with chief executive officers reporting directly to the head of the Department, in line with a general trend towards centralised governance of public health services in Australia. ${ }^{6}$

The CSRP has its origins in the 'Maggie Project' that had been underway in the Hunter region of New South Wales since $2002^{7}$ and the Access Block Improvement Program that commenced at 10 major hospitals in $2004 .^{8}$ Funding for the CSRP was provided for 3 years from July 2005, including the costs of engaging external consultants, backfilling staff participating in redesign projects and undertaking an independent evaluation. The program was funded to operate at three levels, with the Health Services Performance Improvement Branch of the Department providing overall direction, policy development and knowledge management; clinical redesign units in each area health service providing infrastructure and support for redesign projects and, at the service level, redesign projects around specific aspects of care, ranging from relatively small-scale projects in well defined clinical areas to broader initiatives across whole area health services. The CSRP is distinguished by the large-scale of the program and the commitment of significant resources (\$70 million over 3 years). Key elements of the project methodology are summarised in Table 1.

By June 2007, when the research reported here commenced, some 70 separate projects had been initiated. These projects had mainly focussed on improving access to emergency services and surgery, defined by performance targets for treatment in emergency departments, and admission to hospital or elective surgery within recommended time frames. As reported in detail elsewhere, ${ }^{9}$ performance improvements in both clinical areas since mid-2004 are impressive, particularly in light of the pattern of declining performance that had been evident previously and the unprecedented growth in demand during the period. Furthermore, equity of access to both emergency department services and elective surgery improved markedly. The views of senior departmental managers on the specific outcomes they attributed to the CSRP have been published elsewhere. $^{10,11}$

The aim of our research was to explore the views of managers with regard to their experiences of participating in the CSRP and the impact of the Program. It was recognised that the three components of the reform strategy are intertwined and cannot be considered in isolation, necessitating broad ranging interviews that went well beyond the CSRP.

\section{Methods}

Between June and September 2007 a total of 42 senior managers in the Department, area health services and hospitals were interviewed in relation to the CSRP and the broader NSW health reform strategy. The number of departmental interviews was largely determined by inclusion of all key leaders in the reform strategy. Purposive sampling was used to identify potential interviewees from area health services and hospitals to achieve an equivalent number (Table 2). Six people declined to be interviewed and two did not respond to the invitation. The interviews were semi-structured, open-ended and conversational in tone, allowing issues that interviewees wanted to raise to be explored. An interview guide was developed which included topics such as performance management, the influence of additional resources, the nature of behaviour change in senior managers, the use of data and evidence to improve performance, what has been learnt about improving performance, and the role of competition between health services as a driver for change. The last of these - in the form of competition between managers to achieve the performance targets - was included as this was seen by the Department as a key component of their approach to performance management.

With one exception all managers had been working in their current, or equivalent, position within the health system since the commencement of the CSRP (a period of 2 years) and were very experienced. For example, hospital-level managers were from teaching hospitals, major metropolitan hospitals or rural base hospitals, including 11 general managers and 3 directors of nursing. Area-level managers included chief executive officers and directors of portfolios covering clinical operations, performance and clinical governance. In total, 32 managers had a clinical background.

Interviews averaged $65 \mathrm{~min}$ in length, ranging from $39 \mathrm{~min}$ to over 2 hours. Interviews were recorded digitally, except for one instance where the interviewee preferred that the interview was recorded by the taking of notes. All interviews were transcribed. To ensure accuracy of quotations each transcript was compared with the original recording and corrected where possible. Content 
Table 1. Key elements of CSRP projects

\begin{tabular}{ll}
\hline Element & Characteristics \\
\hline Priority setting & $\begin{array}{c}\text { Priorities identified with a 'top down' approach but with an effort to engage key stakeholders and develop } \\
\text { 'bottom up' solutions. } \\
\text { Froject management }\end{array}$ \\
$\begin{array}{l}\text { Formal project management approach with high levels of structure delivered by the expertise and resources } \\
\text { of external consultants. } \\
\text { Strict adherence to tight deadlines, certainly in the initial stages of a project. } \\
\text { Resources to back-fill staff to facilitate participation. }\end{array}$ \\
Strong performance accountability with the use of executive sponsors, short time frames and daily review. \\
Focus on performance (efficiency), with much less emphasis on quality and safety. \\
A focus on process. \\
Using the idea of a patient journey to think beyond the confines of individual departments or services. \\
An 'improvement by objectives' approach. \\
Reliance on data linked to the project key performance indicators (KPIs). \\
Capturing patient experiences. \\
Structuring of new groups to address organisation wide issues. \\
Working in teams.
\end{tabular}

Table 2. External evaluation interviews

\begin{tabular}{lc}
\hline Level & No. interviewed \\
\hline Hospital & 14 \\
Area health service & 13 \\
Health Department & 15 \\
Total & 42 \\
\hline
\end{tabular}

analysis was undertaken using Leximancer document mapping software. Quotations are attributed to interviewees by A (area health service), D (Department) and H (Hospital). The research was approved by the University of Wollongong/Illawarra Area Health Service Human Research Ethics Committee.

\section{Results}

Four key themes were identified in the interviews:

- the nature and extent of the cultural change that is felt to be taking place;

- the important role of senior local leaders;

- the benefits (and otherwise) of performance management systems; and

- the key challenge of sustaining improvements.

\section{Cultural change}

There was agreement amongst interviewees at all levels that there had been an improvement in managers' 'understanding of the business' over the first 2 years of the CSRP, with a stronger orientation towards efficiency, achieving results and using data to support decision-making:

I think people understand their business a lot more ... they're a lot more responsive to changes ... When you know your business and you've got good data and you respond to it in real time, you can actually make a difference. (D)

If we've driven a cultural change, that cultural change of use of data about making informed decisions on good data has been a significant improvement, major improvement.
We're much better informed about what's actually happening in the place, whereas before it was, not so much anecdotal, a bit of gut, a bit of this ... people are thinking practically about what the data's telling us. (H)

Despite this, some managers (particularly at the area and hospital levels) believed that the CSRP on its own would not change the culture of the health system:

You are naive if you think that a project coming into a large organisation with a history of traditions and conventions will lead to a change in that organisation. Other things must occur beyond the project itself to lead to a change in the performance of the organisation, and it's not possible to significantly, at least, separate out performance from culture within the organisation. $(\mathrm{H})$

Interviewees recognised the significant improvements in emergency department and elective surgery performance but also expressed some concern (at all levels) that there has been too much emphasis on certain services, particularly emergency departments, and that this may be resulting in some distortion (tunnel vision) in terms of overall system performance:

We've created a system where the EDs are bottlenecks, we have our principal measures in the bottlenecks and naturally enough those measures are always problematic and we keep throwing more money at the bottlenecks ... People concentrate on what's measured ... What other system do you know that measures itself in terms of the things it doesn't do, rather than the things it does? ... We've got to move away from this absolute obsession with Emergency Departments. (D)

Interviewees expressed a range of views about whether a culture of competition had developed as a result of the focus on performance management and whether this was a healthy development. Some saw competition as a 'tool' that could be useful, but only if done in the 'right way', to promote improvement. The importance attributed to competition appeared to vary between interviewees (and their particular circumstances) and was not a consistent, systemic factor. 
One aspect of competition raised by several interviewees working at the levels of Areas and Hospitals was that no one wanted to be at the bottom of any performance 'league table'. Interestingly, no one wanted to be at the top of such a table either. Achieving targets is more of a motivator than performing better than someone else, as expressed by one hospital general manager:

If you're at the top you're constantly being shown off out there and when you fall, you fall, 'cos we all fall at some point. Even the best will have a bad patch, and they do. The bottom, same sort of thing, you don't want to be at the bottom ... I just want to meet the targets ... if we meet the targets, it means we're getting people through in a very safe, effective way. $(\mathrm{H})$

\section{Leaders}

The importance of local leadership to achieving improvements was referred to repeatedly during the interviews. Those working in area executive positions, and hence well placed to identify the reasons for variations in performance between hospitals, identified leadership at a local level as a key factor:

[Hospital] was our star performer, engaged particularly well with the process, and achieved significant access improvements, through the Emergency Department. I think, in part, that was attributable to a number of things, strong executive management and leadership buy-in we had, and the good working relationship that they developed with the consultants that were engaged ... the point is too, unless you've got some clinician leadership and management to drive the solutions, it's not viable, or not sustainable. (A)

Although many interviewees commented on the need for 'strong' leadership, there was little clarity as to the specific elements of such leadership. For instance, there were no suggestions about the need for leaders to be excellent communicators, the role of the leader in developing people, or to leaders coaching others. One senior manager highlighted a need for 'pretty intensive coaching', but for others, the 'people' skills of leadership did not emerge unprompted. Related to this, many interviewees at the Area level expressed concern about the capability of existing health service managers, an issue that is seen as a systemic problem:

Across the system I think we've underestimated the requirement to focus on capacity building amongst the people that will drive change. We've made a number of assumptions that because you've got to a certain level, then in some way you have got a core skill set and my assessment is that assumption is often not true. (A)

Leadership does not take place in isolation. The range of positions nominated as being sources of 'leadership' for the CSRP (including senior managers within the Department, area chief executives, hospital general managers and clinical leaders) highlighted the importance of having a multi-level system of leadership, rather than relying on individuals in key positions. Some of those we interviewed recognised this:
We made a very clear decision that we were going to . . . put people into the senior management roles who were demonstrating the behaviours, the style, the values that we want as an organisation ... And I think that's hugely powerful and beneficial. (A)

Where interviewees commented on the perceived weakness of local leadership, much of the problem was attributed to the view that many managers started life as clinicians and then progressed to managerial positions without developing or acquiring the specific leadership skills required for their new roles.

\section{Performance management systems}

Across all three levels of managers interviewees agreed that the increased focus on key performance indicators (KPIs) and performance was positive:

I think the areas have accepted that they have a responsibility to perform now. There's been certainly a much stronger focus on the need to deliver levels of performance ... whereas in the past I think there's been an acceptance that we'll do the best we can ... it's been a shift in the culture of the thinking of accountability. (A)

The targets and the information that is now used is much better ... I think it's more professional and we do actually have good data to compare performance and targets that everybody understands and knows. $(\mathrm{H})$

The concerns identified by managers were not so much about performance management per se but the narrowness of the indicators being used to judge performance and how these were applied:

I think we're very focussed on those narrow performance indicators and I guess most of my colleagues would know that there's half a dozen key issues that you really have got to deliver on and they're the very high profile ones. And so we do pay a significant amount of attention to that. In the context of the whole health system, are those the right things to be focussed on? Possibly not. I think the down side is that you may not be investing enough time in other aspects of the business. We're not actually looking at clinical outcomes as robustly as we might. Or we're not looking at financial management as robustly as we might.

There was also concern that the indicators had been selected because they were easy to measure, and not necessarily because they were important:

I think we've tended to gravitate towards what are the things that we are easily able to measure. And that's actually driven what we're counting. And that's wrong. (A)

Nonetheless, the importance of data was confirmed by interviewees, who noted that measurement was becoming more sophisticated. In particular, the use of performance 'dashboards' (a term used to describe a tool that provides information to management in a timely manner on a set of high level indicators in a format designed to aid decision 
making) is now commonplace and seen as a very important advance:

I think information was a real turning point and it was interesting that we kind of gave people a lift and a buzz when they were able to get much more timely information on their performance as well and they owned that a little bit better ... it just seems to be a really powerful change. (A)

I think it's more professional and we do actually have good data to compare performance and targets that everybody understands and knows. So I think it actually works better, people perform better because they know what they're performing to, the targets don't change every day, which they used to. $(\mathrm{H})$

Interviewees responded in various ways to questions about performance management and suggested that a 'push too hard' scenario may be working against achieving the desired level of change. Although not universal, comments supporting this view were made by interviewees at all three levels (Department, Area, Hospital):

There's a constant hammering, it's almost like a squeaky wheel. I wouldn't call that performance management, in effect it comes down to a set of KPIs for the system and everything that is perceived to improve that, gets pushed. (D)

If you take the view that systems respond by being kicked all the time, then you kick them. And lots of managers take that view, there are many who take that view. [Manager X] does [and] is absolutely open about it. You've got to keep the pressure on. (A)

I think people are tired of getting beat about the head. To continually hammer people and think that by yelling at them, by abusing them, by threatening them is going to make a blind bit of difference, it's not. $(\mathrm{H})$

During the course of the interviews there were some references to potential inaccuracies in available data and how data might be distorted, which raises the question of whether an element of 'gaming' is involved, as has occurred in the NHS in the UK. ${ }^{12}$ For some interviewees these concerns led to a lack of confidence in the quality of the reported data and reported outcomes.

\section{Sustaining improvements}

The biggest issue to emerge from the interviews was the challenge of sustaining improvements. No interviewee was confident that CSRP was sufficiently embedded that it would simply keep going on its own. Many senior and middle managers were perceived to be working at a pace and in a way that was not sustainable:

You can see people almost crumbling under it. And I have to say, I do worry. I don't know where this is going to end, because enormous pressure is being put on people, and with very little recognition of what that's doing to them. (D)

Others were concerned that current reforms had not been going for long enough or reached enough people to become the norm:
One of my biggest frustrations is that I believe a sustainable improvement in performance takes time and investment. The penetration (of clinical redesign) within the organisation is still at very embryonic levels, such that I think if all the resources for clinical redesign were pulled out tomorrow, it would struggle to be sustained in any way, shape or form. (A)

Others highlighted the need for constant re-invention, renewal and investment:

For me the issue around a project clinical redesign and hospital management is about the sustainability and the normalisation of ongoing change ... it's about constant innovation, constant change, constant discussion, constant review about performance. $(\mathrm{H})$

Although recognising the strong focus on process improvement, there was concern that there had not been enough emphasis on instilling appropriate behaviours in key people:

It's about alignment of the people, the behaviours, the culture and the processes that support it that actually delivers ... We've focussed on the process part of it, and it's not until we're actually going to change the people side: the culture, the behaviours, the attitudes, and the approach, that we're really going to truly see the sort of level of improvement that we want to see. (A)

In summary, interviewees identified some aspects of cultural change in the way that senior managers tackle issues such as emergency department and elective surgery performance, with improvements in their 'understanding the business' and approach to problem solving. However, they recognised that CSRP, on its own, would not deliver the desired system-wide cultural change. They repeatedly drew attention to the importance of leadership to the long-term success and sustainability of the program. In general, the increased focus on performance was seen as beneficial with no calls for uncoupling 'redesign' from 'performance'. Their major concern was the likelihood that the system could sustain the performance improvements that have been made by simply doing 'more of the same'.

\section{Discussion}

In seeking to place these managerial perceptions of the CSRP in a broader theoretical framework we would argue that much of what we have described above can be explained from the perspectives of institutional ${ }^{13}$ and neo-institutional theory. ${ }^{14}$ Such institutional perspectives generally emphasise the role of social factors rather than economic or efficiency factors in driving organisational action, including external conformity pressures from regulatory bodies or parent organisations, social pressures from other similar organisations, as well as collective, social construction processes. ${ }^{15}$ More specifically, such theories consider the processes by which organisational structures, and the rules, norms and routines within organisations, become established as the accepted and authoritative guidelines for the way things should be done.

In the context of the CSRP, this view of organisations suggests that the behaviour of any one hospital in NSW will be influenced 
by similar organisations as - in order for hospitals to perform effectively within a larger system - they need to establish legitimacy with their peers which often means conforming to the prevailing rules and belief systems. ${ }^{16}$ The managerial perceptions of the value of competition in driving performance improvement illustrate this point. For example, the expressed wish to meet performance targets but to be 'in the middle' rather than striving to be 'at the top' of any performance 'table' suggest more of a desire to conform to expected norms rather than a deep-seated belief in the intrinsic value of such targets. Such a perspective would suggest that some hospitals in NSW implemented management practices associated with the CSRP because they symbolise legitimacy rather than because they necessarily believe they contribute to local priorities. ${ }^{17}$ Legitimacy is conferred on those hospitals that meet narrow targets, whereas other, broader, organisational priorities are not focussed on quite so robustly.

Our findings about local leadership are consistent with contemporary literature on change management and total quality management. For example, 'the connection between such business transformation and leadership is an important and recurring theme' (p. 91). ${ }^{18}$ However, it is not clear whether there is a common understanding of leadership within the NSW health system such as has been defined by the English NHS with its Leadership Qualities Framework. ${ }^{19}$ Support amongst those we interviewed for moving towards 'systems of leadership' is reflected in the literature where there is evidence that 'senior leaders relying only on their own efforts and a directional top-down approach are unlikely to sustain successful continuous improvement, even with formal incentives' (p. 423). ${ }^{20}$

The relative lack of reference in the interviews to the interpersonal qualities of leadership indicates the need for a better understanding of the skills required of leaders in modern healthcare organisations. "Medicine in order to make that transition into effective 21 st century care, must, in addition to integrating technological advances into therapeutics, focus on the human dimension of interpersonal and organisation structures. Coaching is an essential tool in making that transition a reality' (p. 188). ${ }^{21}$

In short, education and development programs are integral to the success and sustainability of improvement programs like $\mathrm{CSRP}^{22}$ - simply put, 'no learning, no change'. Interviewees drew attention time and time again to the importance of leadership to the long-term success and sustainability of redesign. They identified the need for clarity about who is leading a change program, how the different levels of leadership relate and how a change process should be led, emphasising the need for an appropriate leadership style and the importance of role models for others to follow.

Management development should not be overlooked, or seen as less important than leadership development. Improvement projects frequently fail in implementation and this is as much a management issue as a leadership issue. There is some evidence that coaching and mentoring is coming more to the fore in the roles of senior executives working in the NSW health system, ${ }^{23}$ but our findings suggest room for improvement.

The issue of clinicians moving into management is of particular interest. Clinician managers devote most of their time and effort to the management of finances, staff, organisational/institutional issues and customers, with less emphasis on managing quality, data and processes. Their activity is focussed on discourse, persuasion and negotiation, managing a complex set of expectations. ${ }^{24}$ Participation in a process of change is not just a question of developing 'leadership skills' and competencies but of recognising and clarifying their role.

The research evidence suggests that a good deal of performance variability between organisations participating in a common improvement program can be attributed to the extent to which there exists a 'receptive local context for change', a phrase covering not only leadership but also structure, culture and politics. ${ }^{25}$ This can include having a clear strategic vision; a climate conducive to experimentation and risk taking; and the capacity to absorb new knowledge. ${ }^{26}$ Other researchers have identified factors such as cooperative inter-organisation networks; and good relationships between managers and clinicians. ${ }^{27}$ Interviewees indicated areas where the context is already receptive but there are opportunities to make it more so. 'Context' is not a given or simply background noise to a redesign program; it is central and needs to be managed and developed with the same focus and attention as the program itself. ${ }^{9}$

The general agreement amongst those we interviewed that there has been an improvement in managers' 'understanding of the business' prompts the question as to whether this is indicative of a broader cultural change. The evidence base regarding links between culture and healthcare performance is not extensive, is somewhat problematic, but is generally supportive that such links exist. $^{22}$ According to Schein, culture is layered, with observable patterns of behaviour being classified as Level 1, beliefs and values as Level 2 and assumptions as Level 3. ${ }^{28}$ Change may take place at Level 1 whereas deeper beliefs and assumptions (levels 2 and 3 ) remain unchanged. Until new behaviours become embedded and part of daily routine, they may not necessarily influence deeper levels of culture, ${ }^{29}$ reverting over time to behaviours consistent with underlying (and unchanged) assumptions and values.

The leaders of any change program need to be mindful of the possibility of dysfunctional consequences, with the potential to undo good work in other areas, such as tunnel vision (focussing attention on some areas of performance and excluding other important areas that are unmeasured) and the bullying and intimidation of staff in under-performing organisations. ${ }^{29}$

In general there was support amongst those we interviewed for the principle of managing performance by setting targets, with concerns primarily limited to the narrow focus of the indicators and how these are being applied. It is vital that targets are well defined and measure the processes and outcomes that really matter. Care must be taken that targets don't simply become 'frozen ambitions' but are used to promote dialogue and learning. ${ }^{30}$ There is considerable evidence to support the view that setting performance targets may distort behaviour as opposed to leading to genuine improvements. Bottlenecks can be moved to a different part of the system rather than eliminated from the system as a whole.

A target can be achieved by improving the system, distorting the system or distorting the data. There is a risk "that target-driven management subverts sustainable improvement as the short-term 
appeal of target achievement overtakes the desire to bring processes under control' (p. 95). ${ }^{31}$

Centralised performance management can improve coordination and integration, but 'it is unclear whether these benefits outweigh the unintended and dysfunctional consequences of managing organisations in that way'. ${ }^{32}$ The aim should be not only to achieve targets but to understand why and how they have been achieved (or not achieved) 'performance measures can describe what is coming out of the black box of a public agency, as well as what is going in, but they don't necessarily reveal what is happening inside' (p. 592). ${ }^{33}$ There is an extensive and diverse literature on the measurement of performance that summarises characteristic responses to a performance management framework as an evolution with four phases:

- Initial enthusiasm.

- Proliferation of measures and fragmentation of effort.

- Sober reassessment and reflection on the complexity of the task.

- A move towards consensus and identifying solutions. ${ }^{34}$

There were elements of 'initial enthusiasm' amongst those we interviewed but also concern about the large number of current performance indicators, indicating the value of moving to the 'sober reassessment and reflection' and 'consensus and solutions' phases of performance management to consolidate and refine the current system. Performance management to drive change involves a delicate balancing act. Without sufficient push, people 'go to seed' in their comfort zone and avoid change; push too hard and people 'go to ground' and don't change. The ideal is the middle position where people feel the need to strive, have ambition and stay focussed on performance.

The biggest issue to emerge from our interviews - the question of sustainability - was one of the five key risks identified for the CSRP in the original Business Case. It was clear from the interviews that a 'circuit breaker' is required to find ways to continue to improve performance in ways that are sustainable. One proposed 'circuit breaker' is the NSW Health strategy to create $^{35}$ a sustainable framework for 'redesign principles' so that they become widespread across the system.

\section{Conclusions}

Our findings are generally consistent with the literature on change management, performance management and leadership and indicate areas where the reform agenda can be improved and consolidated. This research builds on previous work in NSW that identified support for performance management based on a 'twoway, multilevel approach' that is 'supported by the organisational culture' ${ }^{36}$ Evidence from high-performing healthcare systems around the world indicates the need to invest significantly in leadership-level skills for large-scale change; to mobilise for improvement, strategically align goals, and create measures and implementation initiatives; to work explicitly with models and theories of large-scale change; and to balance short-term operational results with longer term transformation. ${ }^{5}$

Overall, the alignment of clinical redesign and performance management has been instrumental in major changes that are occurring, at least at the management level, within the NSW health system. Cultural change has started to take place, with a shift from process to results, from anecdotes to data and from can't/won't do to can do. But these changes in observed patterns of behaviour will only influence deeper levels of culture if they become embedded and part of daily routine. It is unrealistic to think that CSRP, on its own, will deliver cultural change.

There is concern that changes may not be sustainable as the pressure on managers is excessive. Although there is some evidence of dysfunctional aspects of performance management there is no call either for the focus on performance or the work to redesign the system to be abandoned. Consideration needs to be given to how to consolidate the gains made and build upon them.

There is a consensus in the literature that, although it is possible to measure and improve performance, there are some significant challenges: a need to move to more meaningful and strategic measures of performance, a need to consider the relevance of measures to system change, that performance management is complex and technically challenging, and that implementation requires significant organisational change. ${ }^{37}$ These challenges are echoed in our findings and suggest the need for ongoing work to refine and improve the reform strategy in the health system.

\section{Competing interests}

The authors declare that they have no competing interests.

\section{Acknowledgements}

The evaluation of the Clinical Services Redesign Program was funded by the New South Wales Department of Health.

\section{References}

1 Australian Council for Safety and Quality in Health Care. Patient safety: towards sustainable improvement. Fourth Report to the Australian Health Ministers' Conference. Canberra: Commonwealth of Australia; 2003.

2 Department of Health. High Quality Care for All. NHS Next Stage Review Final Report. London: The Stationery Office; 2008.

3 Institute of Medicine. Crossing the quality chasm: a new health system for the 21st century. Washington, DC: National Academy Press; 2001.

4 Lukas CV, Holmes SK, Cohen AB, Restuccia J, Cramer IE, Schwartz M, Charns MP. Transformational change in health care systems: an organizational model. Health Care Manage Rev 2007; 32: 309-20.

5 Bevan H, Ham C, Plsek PE. The next leg of the journey: How do we make High Quality Care for All a reality? Coventry: NHS Institute for Innovation and Improvement; 2008.

6 Dwyer JM. Australian health system restructuring - what problem is being solved? Aust New Zealand Health Policy 2004; 1: 6. doi:10.1186/ 1743-8462-1-6

7 McDonald B, Swan J, Considine R, Cook J, Lawrence A, Weller D, Reardon J. Evaluation of the Maggie Program: a journey in health services improvement. Hunter New England Area Health Service; 2006.

8 Duckett S, Dwyer J, Cunningham J, Leggat S, Jackson T, Cranny C, Cameron P. Access Block Improvement Program Evaluation: Final Report. La Trobe Health Management Group; 2005.

9 Masso M, Marcolin S, Lago L, Siminski P, Quinsey K, Hasan H, McCarthy G, Robert G, Bate P, Eagar K. Second Annual Report on the NSW Clinical Services Redesign Program (CSRP). Wollongong: Centre for Health Service Development, University of Wollongong and University College London; 2007.

10 McGrath KM, Bennett DM, Ben-Tovim DI, Boyages SC, Lyons NJ, O'Connell TJ. Implementing and sustaining transformational change in health care: lessons learnt about clinical process redesign. Med J Aust 2008; 188: S32-5. 
11 O'Connell TJ, Ben-Tovim DI, McCaughan BC, Szwarcbord MG, McGrath KM. Health services under siege: the case for clinical process redesign. Med J Aust 2008; 188: S9-13.

12 Bevan G, Hood C. What's measured is what matters: targets and gaming in the English Public Health Care System. Public Adm 2006; 84: 517-38.

13 Greenwood R, Hinings CR. Understanding radical organizational change: bringing together the old and the new institutionalism. Acad Manage Rev 1996; 21: 1022-54. doi:10.2307/259163

14 Powell WW, DiMaggio PJ. The new institutionalism in organizational analysis. Chicago: University of Chicago Press; 1991.

15 Westphal JD, Gulati R, Shortell SM. Customization or conformity? An institutional and network perspective on the content and consequences of TQM adoption. Adm Sci Q 1997; 42: 366-94. doi:10.2307/2393924

16 Scott WR, Christensen SM (Eds). The institutional construction of organizations: international and longitudinal studies. Thousand Oaks, CA: Sage; 1995.

17 Katz-Navon T, Naveh E, Stern Z. The moderate success of quality of care improvement efforts: three observations on the situation. Int $J$ Qual Health Care 2006; 19: 4-7. doi:10.1093/intqhe/mz1058

18 Hoyte DS, Greenwood RA. Journey to the North face: a guide to business transformation. Academy of Strategic Management Journal 2007; 6: 91-104.

19 NHS Institute for Innovation and Improvement. NHS Leadership Qualities Framework. 2006. Available at http://www.nhsleadership qualities.nhs.uk/ [verified 3 July 2010].

20 Øvretveit J. Leading improvement. J Health Organ Manag 2005; 19: 413-30. doi:10.1108/14777260510629661

21 Henochowicz S, Hetherington D. Leadership coaching in healthcare. Leadersh Organ Dev J 2006; 27: 183-9. doi:10.1108/0143773061 0657703

22 Bate P, Mendel P, Robert G. Organising for quality, the improvement journeys of leading hospitals in Europe and the United States. Oxford: Radcliffe Publishing; 2007.

23 Liang Z. Characteristics, competencies and challenges: a quantitative and qualitative study of the senior executive workforce in New South Wales, 1990-1999. Brisbane, QLD: Griffith University; 2006.

24 Braithwaite J. An empirically-based model for clinician-managers' behavioural routines. J Health Organ Manag 2004; 18: 240-61. doi:10.1108/14777260410554269

25 Bate P, Robert G, McLeod H. Report on the 'Breakthrough' Collaborative approach to quality and service improvement within four regions of the NHS. A research based investigation of the Orthopaedic Services Collaborative within the Eastern, South and West, South East and Trent regions. Research Report no. 42. 2002. Available at http://www.hsmc.bham.ac.uk/publications/Archive.shtml\# 2002 [verified 3 July 2010].
26 Greenhalgh T, Robert G, Macfarlane F, Bate P, Kyriakidou O. Diffusion of innovations in service organizations: systematic review and recommendations. Milbank $Q$ 2004; 82: 581-629. doi:10.1111/j.0887378X.2004.00325.x

27 Pettigrew AM, Ferlie E, McKee L. Shaping strategic change. London: Sage; 1992.

28 Schein EH. Organisational culture and leadership. 2nd edn. San Francisco: Jossey-Bass; 1992.

29 Mannion R, Davies HTO, Marshall M. Cultures for performance in health care. Maidenhead, UK: Open University Press; 2005.

30 Van Der Knaap P. Responsive evaluation and performance management. Evaluation 2006; 12: 278-93. doi:10.1177/1356389006069135

31 Walley P, Silvester K, Mountford S. Health-care process improvement decisions: a systems perspective. Int J Health Care Qual Assur Inc Leadersh Health Serv 2006; 19: 93-104.

32 National Institute for Health Research Service Delivery and Organisation. Achieving high performance in health care systems: the impact and influence of organisational arrangements: NHS Service Delivery and Organisation R\&D Programme; 2006. Available at http://www.sdo.nihr. ac.uk/files/adhoc/55-briefing-paper.pdf [verified 3 July 2010].

33 Behn RD. Why measure performance? Different purposes require different measures. Public Adm Rev 2003; 63: 586-606. doi:10.1111/ 1540-6210.00322

34 Adair CE, Simpson L, Birdsell JM, Omelchuk K, Casebeer A, Gardiner HP, Newman S, Beckie A, Clelland S, Hayden KA, Beausejour P. Performance Measurement Systems in Health and Mental Health Services: models, practices and effectiveness. A state of the science review. 2003. Available at http://www.ahfmr.ab.ca/grants/docs/state of_science_reviews/Adair_Review.pdf [verified 3 July 2010].

35 Brown J. Strategy for the sustainability of the Clinical Services Redesign Program. Sydney: NSW Health; 2007.

36 Liang Z, Howard PF. Views from the executive suite: lessons from the introduction of performance management. Aust Health Rev 2007; 31: 393-400. doi:10.1071/AH070393

37 Adair CE, Simpson E, Casebeer AL, Birdsell JM, Hayden KA, Lewis S. Performance measurement in healthcare: part II - state of the science findings by stage of the performance measurement process. Health Policy 2006; 2: 53-78.

Manuscript received 6 November 2008, accepted 22 October 2009 
Reproduced with permission of the copyright owner. Further reproduction prohibited without permission. 